\title{
Phenotypic Detection of Carbapenem Resistance among Escherichia coli and Klebsiella Isolates Obtained from Various Clinical Samples
}

\author{
Jaspal Kaur*, Priyanka Khanna, Sheevani and Kailash Chand \\ Punjab Institute of Medical Sciences Jalandhar, Punjab, India \\ *Corresponding author
}

\begin{abstract}
Keywords
Carbapenemase,

E.coli, Klebsiella, KPC, MBL.

Article Info

Accepted:

14 September 2017

Available Online:

10 October 2017 require a simple and inexpensive testing method. A total of 250 isolates which included Escherichia coli (113) and Klebsiella spp (137) were screened for meropenem resistance by Kirby-Bauer disc diffusion method. Modified Hodge test (MHT) and a confirmatory phenotypic detection test was done by using combined discs of meropenem alone and with those of inhibitor phenylboronic acid (PBA), EDTA and both PBA and EDTA, for the detection of carbapenemase production and differentiation of KPC and MBL enzymes. 49 $(19.6 \%)$ isolates showed reduced susceptibility to meropenem. Modified Hodge test was positive in $41(83.7 \%)$ of carbapenem resistant isolates while confirmatory phenotypic detection test showed $69.4 \%$ of carbapenem resistant isolates positive for carbapenemase production. MBL activity was observed in $16(6.4 \%)$ of the total 250 clinical isolates and KPC production was seen in $8(7.2 \%)$. Fifteen of the total 49 CRE isolates were negative for MBL and KPC production. None of the isolates showed co-production of KPC and MBL enzymes. Our study emphasises on the need of a simple and reliable method for phenotypic detection of MBL and KPC carbapenemases for clinical as well as epidemiological purposes.
\end{abstract}

\author{
A B S T R A C T
}

Acquired resistance to carbapenems mediated by carbapenemases in gram-negative pathogens such as Escherichia coli and Klebsiella has become worldwide. Its detection is of utmost importance in deciding the most appropriate therapeutic regimen and for this we

\section{Introduction}

Carbapenems are broad spectrum beta-lactam antimicrobial agents, stable to hydrolysis by most beta- lactamases. Their introduction in 1980 was a major advancement in medical field as they were used as last resort antibiotics for treating infections due to multidrug resistant organisms (Yong et al., 2009). But unfortunately acquired resistance to carbapenems in Gram-negative pathogens such as Enterobacteriaceae has now become a worldwide problem. This resistance is mediated by carbapenemases such as Ambler class B metallo-_beta-lactamases (MBL), including IMP, VIM, and NDM, as well as by plasmid mediated clavulanic acid inhibited class A beta-lactamases such as Klebsiella pneumoniae carbapenemase (KPC) and Guiana extended spectrum (GES) and the class D beta-lactamase OXA-48 (Queenan et al., 2007). These enzymes are usually encoded by mobile DNA elements and have a high capacity for dissemination. Infections due to these resistant strains are associated with higher morbidity and mortality as it 
severely limits treatment options. Carbapenemase producing strains are resistant not only to carbapenems but to almost all beta-lactam antibiotics. Moreover, carbapenem resistance in Enterobacteriaceae is often associated with extended-spectrum beta-lactamase (ESBL) or with AmpC betalactamase production and porin loss.

Polymyxins, tigecycline, and less frequently aminoglycoside antibiotics are the treatment options for carbapenemase producing bacteria based on in vitro susceptibility (Arnold et al., 2011). Hence accurate and timely detection of these resistant mechanisms is very important in deciding the appropriate treatment. But detection of the resistant mechanisms is always a serious challenge to the clinical laboratories.

Molecular methods which are gold standard for their detection are available in only a few reference laboratories. Hence we carried out phenotypic tests i.e. Modified Hodge test (MHT) and other tests based on the synergy between MBL or KPC inhibitors and carbapenems to detect carbapenemase production in Escherichia coli and Klebsiella pneumoniae isolates obtained from various clinical samples.

\section{Materials and Methods}

The study was conducted in the Department of Microbiology, Punjab Institute of Medical Sciences, Jalandhar, India. A total of 250 isolates which included Escherichia coli (113) and Klebsiella spp. (137) obtained over a period of six months from July 2016 to December 2016 from various clinical specimens such as pus, urine, sputum, blood, pleural fluid, ascitic fluid, endotracheal aspirate, stool and vaginal swab of hospitalized patients were studied. The isolates were identified by standard microbiological techniques.

\section{Antimicrobial susceptibility testing}

The isolates were tested for antimicrobial susceptibility by disc diffusion method according to CLSI guidelines. The following antibiotics were used; cefotaxime $(30 \mu \mathrm{g})$, cefpodoxime $(30 \mu \mathrm{g})$, ceftriaxone $(30 \mu \mathrm{g})$,

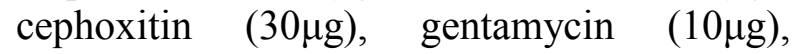

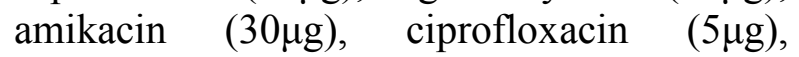
norfloxacin $(10 \mu \mathrm{g})$, nitrofurantoin $(100 \mu \mathrm{g})$, cotrimoxazole $(25 \mu \mathrm{g})$, piperacillin/tazobactam $(100 / 10 \mu \mathrm{g})$, meropenem $(10 \mu \mathrm{g})$, imipenem $(10 \mu \mathrm{g})$, tigecycline $(15 \mu \mathrm{g})$, polymyxin $\mathrm{B}$ (300 units), colistin $(10 \mu \mathrm{g})$. All the antibiotic discs were procured from Hi-media, Mumbai.

\section{Phenotypic screening for carbapenemase production}

Carbapenemase production was screened by disc diffusion; all the isolates with a reduced susceptibility to meropenem (diameter of zones of inhibition, $\leq 21 \mathrm{~mm}$ ) were considered as screen positive and further screened for carbapenemase production by using Modified Hodge test. A combined disc test was performed as a confirmatory phenotypic test.

\section{Modified Hodge test}

The strains were subjected to Modified Hodge test for detection of carbapenemases as per recommendation of Clinical and Laboratory Standards Institute (CLSI) guidelines, 2011 (CLSI, 2011). An overnight culture suspension of Escherichia coli ATCC 25922 adjusted to $0.5 \mathrm{McFarland}$ standard was inoculated using a sterile cotton swab on the surface of a Mueller-Hinton agar. After drying, $10 \mu \mathrm{g}$ meropenem disk was placed at the centre of the plate and the test strains were streaked from the edge of the disk to the periphery of the plate in four different directions. The plate was incubated overnight at $37^{\circ} \mathrm{C}$. The presence of a cloverleaf shaped zone of inhibition due to carbapenemase 
production by the test strains was considered as positive.

\section{Confirmatory phenotypic test}

A confirmatory phenotypic detection test was done by using combined discs of meropenem alone and with those of inhibitor phenylboronic acid (PBA), EDTA and both PBA and EDTA, for the detection of carbapenemase production and differentiation of KPC and MBL enzymes.

The stock solution of PBA was prepared by dissolving phenyl boronic acid (SigmaAldrich, Germany) in DMSO at a concentration of $20 \mu \mathrm{g} / \mathrm{ml}$. $20 \mu \mathrm{L}$ of the stock solution (containing $400 \mu \mathrm{g}$ of PBA) was dispensed onto commercially available meropenem discs (Hi-media). The stock solution of EDTA (Sigma-Aldrich, Germany) was prepared by dissolving anhydrous EDTA in distilled water at a concentration of $0.1 \mathrm{M}$. From this solution, $10 \mu \mathrm{L}$ (containing $292 \mu \mathrm{g}$ of EDTA) was dispensed onto Meropenem discs. The discs were dried and used within 60 minutes. The test was performed by inoculating the test organism on Mueller Hinton Agar and placing one disc of meropenem without any inhibitor and three discs of meropenem, each containing $400 \mu \mathrm{g}$ of PBA, 292 $\mu \mathrm{g}$ of EDTA and one disc containing both i.e. $400 \mu \mathrm{g}$ of PBA and $292 \mu \mathrm{g}$ of EDTA on it. The agar plates were incubated at $37^{\circ} \mathrm{C}$ overnight. The diameter of the growth inhibitory zone seen around the meropenem disc with PBA, EDTA, PBA+EDTA was compared with that seen around the plain meropenem disc (Tsakris A et al., 2010).

\section{Interpretation}

Production of KPC was considered when the growth inhibitory zone diameters seen around the meropenem disc with PBA and the meropenem disc with PBA+EDTA has increased to $>=5 \mathrm{~mm}$ as compared to the growth inhibitory zone diameter seen around the disc containing meropenem alone.

Production of MBL was considered when the growth inhibitory zone diameters seen around the meropenem disc with EDTA and the meropenem disc with PBA+EDTA had increased to $>=5 \mathrm{~mm}$ as compared to the growth inhibitory zone diameter seen around the disc containing meropenem alone.

Production of both KPC and MBL enzymes was considered when the growth-inhibitory zone diameter around the meropenem disk with both PBA and EDTA was increased >=5 $\mathrm{mm}$ compared with the growth-inhibitory zone diameter around the disk containing meropenem alone while the growth inhibitory zone diameters around the meropenem disk with PBA and the meropenem disc with EDTA were increased $<5 \mathrm{~mm}$ compared with the growth-inhibitory zone diameter around the disk containing meropenem alone (Tsakris et al., 2010).

\section{Results and Discussion}

Out of total of 250 isolates of Escherichia coli (113) and Klebsiella spp.(137),49 (19.6\%) isolates were carbapenem resistant These included $15(13.3 \%)$ strains of $E$. coli and $34(24.8 \%)$ strains of Klebsiella spp. Majority of the carbapenem resistant strains were isolated from endotracheal secretions of ICU patients and blood samples of NICU patients (Table 1). All the carbapenem non-susceptible isolates were multidrug resistant with 80$100 \%$ resistance to aminoglycosides, fluoquinolones and cephalosporins. One carbapenem resistant E. coli and two carbapenem resistant Klebsiella were resistant even to colistin and polymyxin B (Figure 1). Modified Hodge test showed 41(83.7\%) of $\mathrm{CRE}$ to be positive for carbapenemase 
production (Figure 2). Confirmatory phenotypic detection test done by using combined discs of meropenem alone and with those of inhibitor phenylboronic acid (PBA), EDTA and both PBA and EDTA, showed MBL activity in 16(6.4\%) isolates (i.e., $7 \mathrm{E}$. coli and 9 Klebsiella spp.) and KPC production in $18(7.2 \%)$ (i.e., 6 E. coli and 12 Klebsiella spp.) (Figure 3). Fifteen of the total $49 \mathrm{CRE}$ isolates were negative for MBL and KPC production. None of the isolates showed co-production of KPC and MBL enzymes.
Carbapenem- resistant Enterobacteriaceae (CRE) is feared as new superbug. Tom Frieden, former head of the Centers for Disease Control and Prevention has referred to CRE as nightmare bacteria.

They are primarily nosocomial infectious agents. Risk factors include mechanical ventilation, underlying immunocompromised state, prolonged hospital stay with exposure to antimicrobials (Patel et al., 2008, Nadkarni et al., 2009).

Table.1 Sample wise distribution of various carbapenem resistant E. coli and Klebsiella isolates

\begin{tabular}{|l|l|l|}
\hline Nature of sample & $\begin{array}{l}\text { Number of isolates } \\
(\mathrm{n}=49)\end{array}$ & Percentage \\
\hline Endotracheal secretions & 21 & 42.8 \\
\hline Sputum & 9 & 18.4 \\
\hline Blood & 7 & 14.3 \\
\hline Pus & 5 & 10.2 \\
\hline Urine & 3 & 6.1 \\
\hline Others & 4 & 8.2 \\
\hline
\end{tabular}

Fig.1 Resistance pattern of carbapenem resistant and non- carbapenem resistant $E$. coli and Klebsiella isolates to various antimicrobial agents

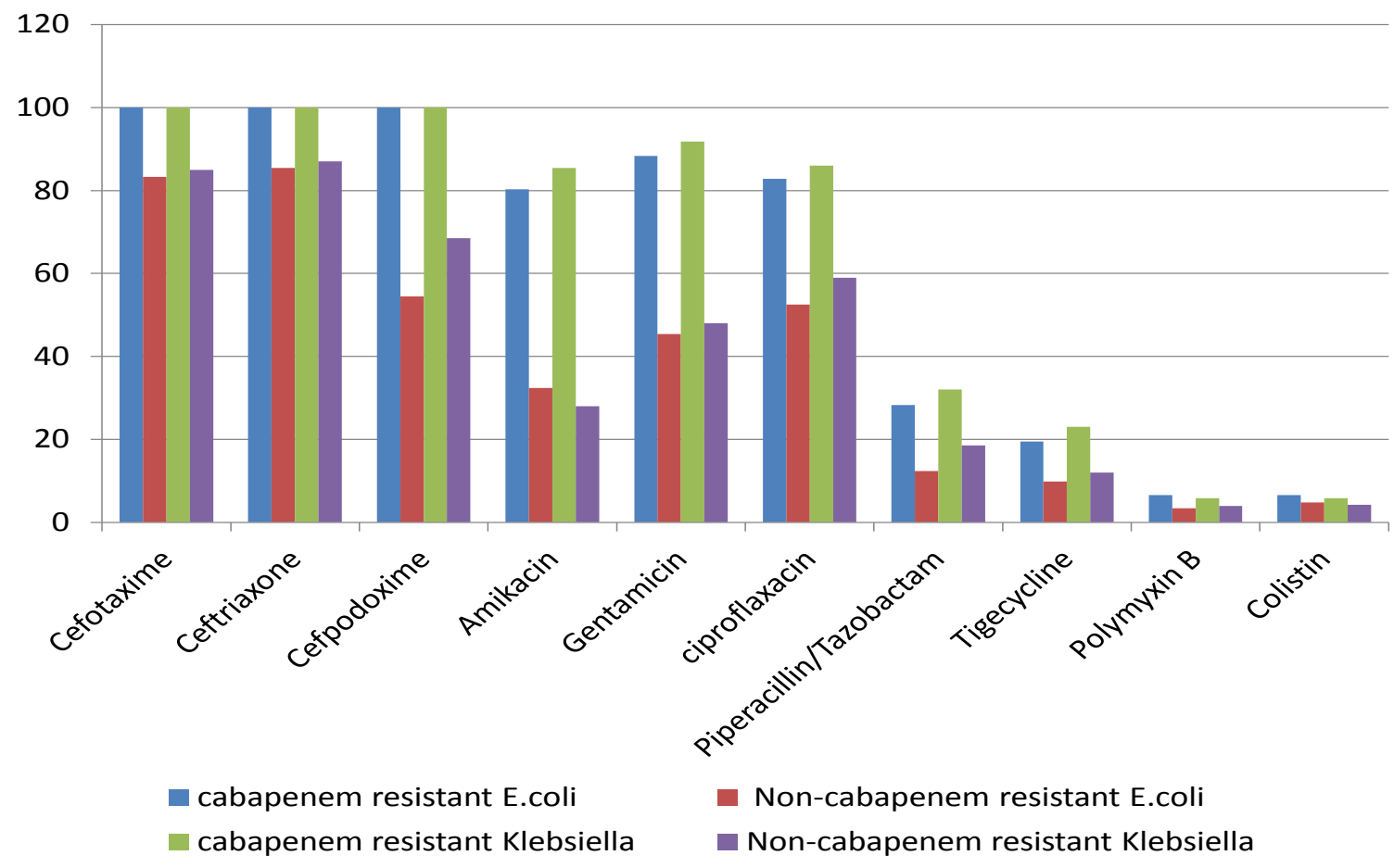


Fig.2 Positive Modified Hodge test showing cloverleaf shaped zone of inhibition around meropenemd

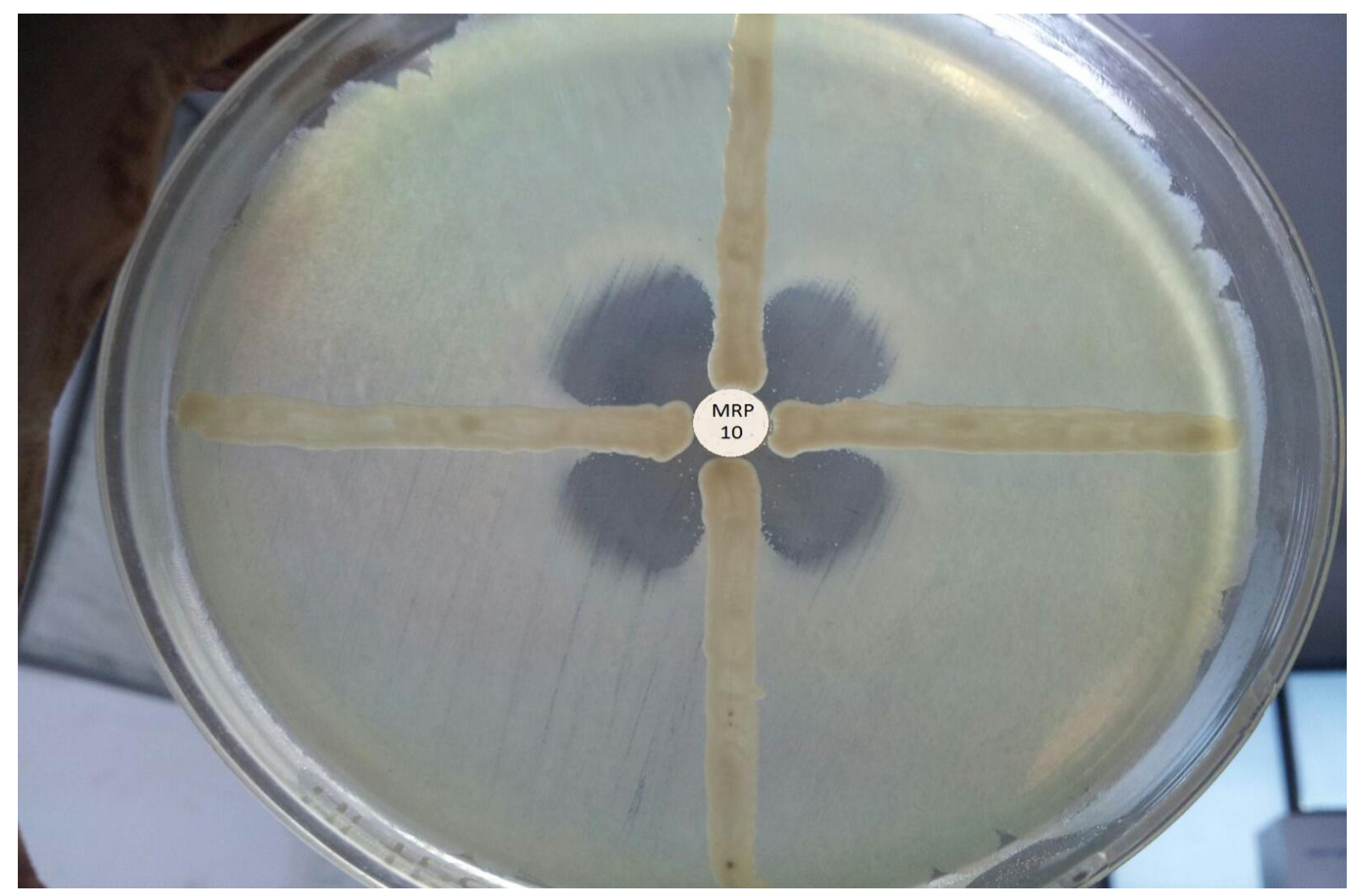

Fig.3 Combined disc test showing MBL production in $E$. coli isolate with enhanced zone $(>5 \mathrm{~mm})$ around meropenem disc with EDTA incorporated in it as compared to meropenem alone

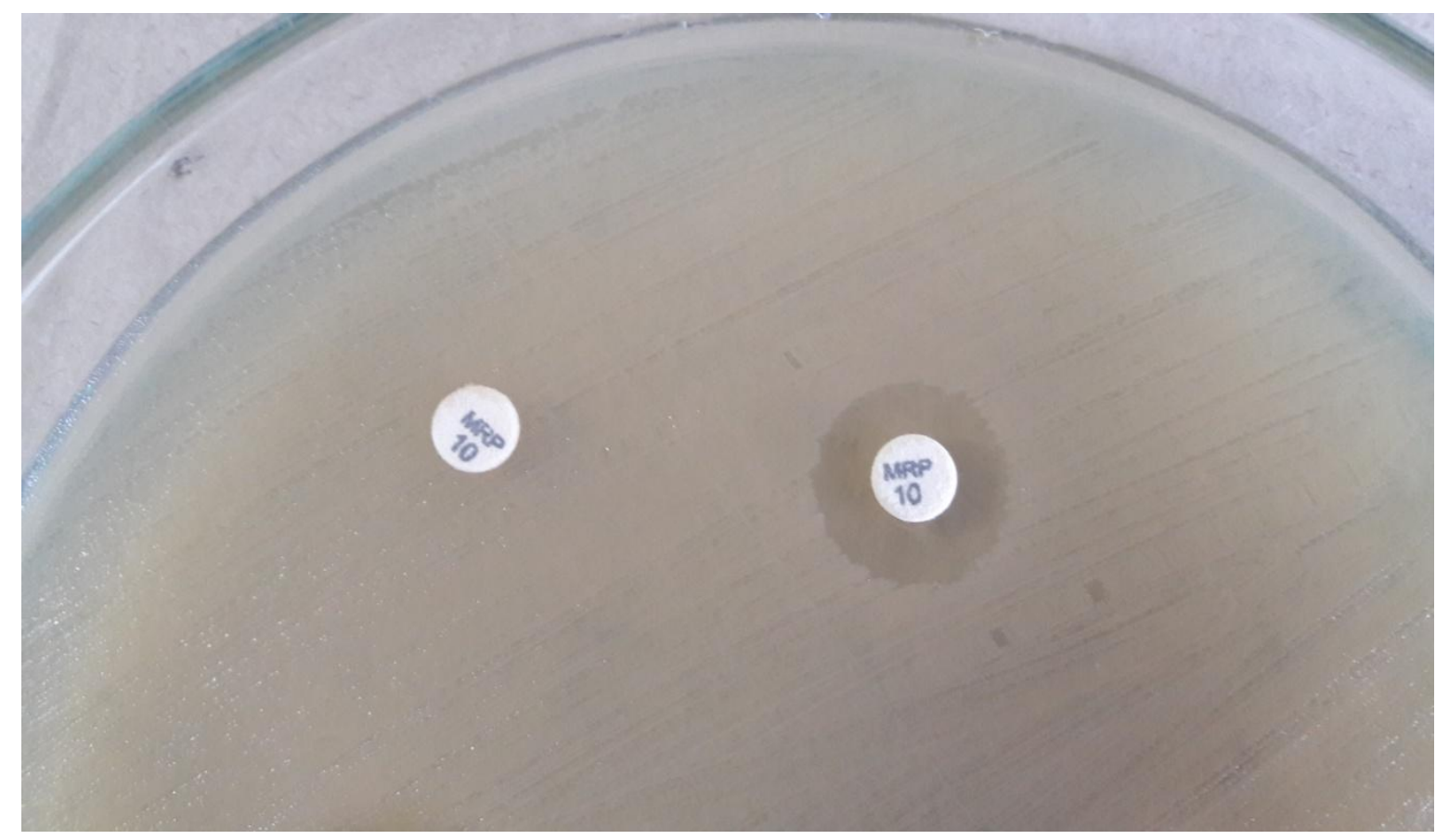


Cabapenem resistance of $5-50 \%$ has been reported in different Indian studies (Datta et al., 2012, Wattal et al., 2010, Chauhan et al., 2015). Prevalence rate in our institution from indoor patients was $19.6 \%$. Reports of $60.5 \%$ carbapenem-resistant Klebsiella pneumoniae from Greece, $28.8 \%$ in Italy and $62.7 \%$ CRE from Egypt shows that the situation is critical worldwide (Hrabak et al., 2014, Fattouh et al., 2015). Hence simple, inexpensive and accurate laboratory method to detect carbapenemase production in clinical isolates of Enterobacteriaceae is helpful, particularly in countries where multi-drug resistant strains are increasingly reported (Nordmann et al., 2012, Schwaber and Carmeli; 2014). Modified Hodge test fulfils the first two criteria but it is not very accurate as it gives false positive results in other beta- lactamases having some marginal carbapenemase activity such as AmpC and ESBLs (Carvalhaes et al., 2010) and cannot distinguish carbapenemase type. In our study Modified Hodge test showed $83.7 \%$ of carbapenem resistant strains positive for carbapenemase production while confirmatory phenotypic detection test done by using combined discs of meropenem alone and with inhibitors like PBA and EDTA showed $69.4 \%$ of carbapenem resistant strains positive for carbapenemase production.

In other cases of carbapenem resistance strains where both tests were negative, the reason could be some other mechanism of resistance like porin loss or hyperproduction of AmpC. Higher positivity shown by modified hodge test could be due reasons mentioned earlier. Chauhan etal in their study detected more cases of carbapenemase production using combined disc test (58.44\% in E. coli and $52.94 \%$ in Klebsiella) than with modified Hodge test $(41.56 \%$ in E.coli and $47.06 \%$ in Klebsiella) (Chauhan et al., 2015). But despite the problems with the interpretation of the modified Hodge test giving relatively high rates of false-positive and false-negative results with some isolates, the CLSI has proposed this test for confirmation of putative carbapenemase producers. However, the modified Hodge test should not be used for final confirmation of carbapenemase production (Hrabak et al., 2014).

Phenotypic detection of MBL producers is based on the specific inhibition of MBLs by chelating agents, most commonly EDTA. According to a recommendation published by EUCAST and European Society of Clinical Microbiology and Infectious Disease (ESMCID) class B enzyme is suspected when a difference of $5 \mathrm{~mm}$ in zone diameter is observed between meropenem 10ug and meropenem plus EDTA0.25M. E test strips containing meropenem and meropenem plus EDTA have high sensitivity and specificity for initial characterization of MBL producing Enterobacteriaceae. (Girlich et al., 2013) Boronate based tests show high sensitivity in detection of KPC producers and the results have been confirmed by molecular tests (Tsakaris et al., 2009, Girlich et al., 2012). Recently hospital infections by Klebsiella pneumonia coproducing KPCs and MBLs have been described (Miriagou et al., 2013). Owing to global spread of carbapenem resistant bacteria, it becomes necessary that all clinical laboratories have method for their detection. Reliable methods like PCR, Carba NP test and MALDI-TOF MS hydrolysis require experienced laboratory personnel and equipment (Hrabak et al., 2014).

So in this situation our study emphasises on the use of simple, cost effective, sensitive and technically less demanding methods to both screen for and confirm the presence of carbapenemses so that timely measure can be taken to curtail its spread as part of infection control practices and also serve as guidance in treatment of cases. So, to conclude combined disc test using meropenem with inhibitors like 
EDTA and boronic acid satisfactorily fulfils this criteria.

\section{References}

Arnold, R.S., Thom, K.A., Sharma, S., Phillips, M., Johnson, J.K. and Morgan, D.J. (2011). Emergence of Klebsiella pneumoniae Carbapenemase (KPC)Producing Bacteria. South Med. J; 104(1): 4045.

Carvalhaes, C.G., Picao, R.C., Nicoletti, A.G., Xavier, D.E. and Gales, A.C. 2010. Cloverleaf test (modified Hodge test) for detecting carbapenemase production in Klebsiella pneumoniae: be aware of false positive results. $J$ Antimicrob Chemother ; 65: 249-251.

Chauhan, K., Pandey, A., Asthana, A.K. and Madan, M. 2015. Evaluation of phenotypic tests for detection of Klebsiella pneumoniae carbapenemase and metallo-beta-lactamase in clinical isolates of Escherichia coli and Klebsiella species. Indian $J$ Pathol Microbiol ;58:31-5.

Clinical and Laboratory Standards Institute. Performance Standards for Antimicrobial Susceptibility Testing: Twenty First Informational Supplement M100-S21. CLSI, Wayne, PA: USA; 2011.

Datta, P., Gupta, V., Shivani, G. and Chander J. 2012. Phenotypic method for differentiation of carbapenemases in Enterobacteriaceae: Study from North India. Indian $J$ Pathol Microbiol ;55(3):357-360.

Fattouh, M., El-din, A.N. and Omar, M.A. 2015. Detection of Klebsiella pneumoniae Carbapenemase (KPC) Producing Gram negative superbugs: An emerging cause of multidrugresistant infections in general surgery department of Sohag University Hospital,

Egypt.
Int.J.Curr.Microbiol.App.Sci ; 4(5): 115

Girlich, D., Halimi, D., Zambardi, G. and Nordmann P.2013. Evaluation of Etest MBL strips for detection of KPC and metallo-carbapenemases in Entero bacteriaceae. Diagn Microbiol Infect Dis ; 77: 200-201.

Girlich, D., Poirel, L., and Nordmann, P. 2012. Value of the modified Hodge test for detection of emerging carbapenemases in Enterobacteriaceae. J Clin Microbiol; 50(2): 477479- 9.

Hrabak, J., Chudackova, E., and Papagiannitsis, C.C. 2014. Detection of carbapenemases in Enterobacteriaceae: a challenge for diagnostic microbiological laboratories. Clin Microbiol Infect ; 20: 839-853.

Miriagou, V., Tzelepi, E., Kotsakis, S.D., Daikos, G.L, Casal, J.B. and Tzouvelekis LS.2013. Combined disc methods for the detection of KPCand/or VIM-positive Klebsiella pneumoniae: improving reliability for the double carbapenemase producers Clin Microbiol Infect ; 19: E412-E415 10.1111/1469-0691.

Nadkarni, A.S., Schliep,T. and Khan, L. 2009. Cluster of bloodstream infections caused by KPC-2 Carbapenemaseproducing Klebsiella pneumoniae in Manhattan. Am J. Infect. Control; 37: 121- 126.

Nordmann, P., Poirel, L. and Dortet, L. 2012. Rapid detection of carbapenemaseproducing Enterobacteriaceae. Emerg Infect Dis; 18: 1503-1507.

Patel, G., Huprikar, S and Factor, S.H. 2008. Outcomes of carbapenem-resistant Klebsiella pneumoniae infection and the impact of antimicrobial and adjunctive therapies. Infect. Control Hosp.Epidemiol.; 29: 1099-1106.

Queenan, A.M.and Bush, K. 2007. Carbapenemases: the versatile beta- 
lactamases. Clin Microbiol Rev; 20:440 e58

Schwaber, M.J. and Carmeli, Y. 2014.An ongoing national intervention to contain the spread of carbapenem-resistant Enterobacteriaceae. Clin Infect Dis; 58: 697-703.

Tsakris, A., Kristo, I. and Poulou,A. 2009. Evaluation of boronic acid disk tests for differentiating KPC-possessing Klebsiella pneumoniae isolates in the clinical laboratory. J Clin. Microbio; 47: 362-367.

Tsakris, A., Poulou, A., Pournaras, S., Voulgari, E., Vrioni, G., ThemeliDigalak, K., Dimitra Petropoulou, D. and Sofianou D. 2010. A simple phenotypic method for the differentiation of metallo beta- lactamases and class A KPC carbapenemases in Enterobacteriaceae clinical isolates. $J$. Antimicrob.Chemother; 65: 1664- 1671. Wattal, C., Goel, N., Oberoi, J.K., Raveendran, R., Datta, S. and Prasad, K.J. 2010.Surveillance of multidrugresistant organisms in tertiary care hospital in Delhi, India. $J$ Assoc Physicians India ; 58:32-36.

Yong, D., Toleman, M.A., Giske, C.G., Cho, H.S., Sundman, K., Lee, K. and Walsh, T.R. 2009. Characterization of a new metallo-beta- lactamase gene, blaNDM1 , and a novel erythromycin esterase gene carried on a unique genetic structure in Sequence Type 14 from India. Antimicrob. Agents Chemother; 53: 5046- 5054.

\section{How to cite this article:}

Jaspal Kaur, Priyanka Khanna, Sheevani and Kailash Chand. 2017. Phenotypic Detection of Carbapenem Resistance among Escherichia coli and Klebsiella Isolates Obtained from Various Clinical Samples. Int.J.Curr.Microbiol.App.Sci. 6(10): 1566-1573. doi: https://doi.org/10.20546/ijcmas.2017.610.188 